\title{
Multiplexing 8 colors with 12 antibodies in a single lymphoid screening tube by flow cytometry for evaluating suspected chronic lymphoproliferative disorders (CLPD)
}

\author{
Rajesh Kumar Bhola ${ }^{1}$ (D) Prabodh Kumar Das ${ }^{2} \cdot$ Sarita Pradhan $^{1} \cdot$ Kaushambi Chakraborty $^{1} \cdot$ Debahuti Mohapatra $^{1}$. \\ Priyanka Samal $^{3}$. Pritish Chandra Patra ${ }^{3}$. Soumya Surat Panda ${ }^{4}$. Sourav Kumar Mishra ${ }^{4}$
}

Received: 11 June 2019 / Accepted: 15 October 2019/Published online: 2 December 2019

(C) Springer-Verlag GmbH Germany, part of Springer Nature 2019

\begin{abstract}
The diagnosis of chronic lymphoproliferative disorder (CLPD) or non-Hodgkin Lymphoma (NHL) is based on the detection of the abnormal clonal lymphoid cells. The flow cytometry (FCM) immunophenotyping not only plays an essential role in the screening of CLPD but also helps in the specific identification and characterization of the expanded aberrant lymphocytes. Over decades, it has evolved from a single parameter to multi-parameter assessment by 3- to 12-color FCM. The greatest challenge is to characterize abnormal lymphoid cells by a limited immunophenotype (IPT) panel. A study was undertaken to evaluate the diagnostic usefulness of a single lymphoid screening tube (LST) FCM assays that included a multiplex of 12 antibody cocktails consisting of CD45, CD19, CD3, CD4, CD8, TCR $\gamma \delta, \mathrm{CD} 5, \mathrm{CD} 20, \mathrm{CD} 56, \mathrm{CD} 38$, kappa, and lambda in an 8-color labeling based on the proposed EuroFlow (EF) antibody panels with slight modifications. We have included the same set of markers and fluorochrome combinations except for CD45 V500-C, CD4 V450, and CD20 V450 instead of CD45 Pacific Orange (PacO), CD20 Pacific Blue (PacB), and CD4 PacB. A logical gating strategy was used to separate different subsets of lymphoid populations for the detection of the clonal lymphoid cells. Further, antibody cocktails were used depending upon the nature of the clonal population like B, T, or natural killer (NK) cells for definitive classification. The results were further correlated with trephine biopsy, immunohistochemistry (IHC), and molecular or cytogenetics wherever required for definitive characterization. A total of 82 consecutive samples with suspicion of CLPD or NHL were analyzed by a 3-laser 8-color flow cytometry. Out of which 73 cases were diagnosed to be CLPD, 2 cases were diagnosed as acute lymphoblastic leukemia (ALL), and 7 cases had polyclonal lymphoid population. We could achieve a diagnostic sensitivity of 100\% in the diagnosis of CLPD. In addition, the average number of tubes as well as antibodies was reduced by one-third in comparison to a standard protocol. The development of 8-color and 12-antibody LST by flow cytometry can provide maximum IPT information and meet economical requirements especially in developing countries by reducing the total cost of antibodies, labor, and time.
\end{abstract}

Keywords Lymphoid screening tube (LST) · Flow cytometry · CLPD

Rajesh Kumar Bhola

raeshkumarbhola@soa.ac.in; rajeshbhola1980@gmail.com

1 Laboratory Haematology Division, Department of Pathology, IMS \& SUM Hospital, Siksha 'O' Anusandhan (Deemed to be University), Bhubaneswar, Odisha 751003, India

2 Department of Medical Oncology/Hematology, All India Institute of Medical Sciences (AIIMS), Bhubaneswar, Odisha, India

3 Department of Haematology, IMS \& SUM Hospital, Siksha 'O' Anusandhan (Deemed to be University), Bhubaneswar, Odisha, India

4 Department of Medical Oncology, IMS \& SUM Hospital, Siksha 'O' Anusandhan (Deemed to be University), Bhubaneswar, Odisha, India

\section{Introduction}

Chronic lymphoproliferative disorders (CLPD) or nonHodgkin lymphomas (NHL) are clinically, morphologically, and immunologically heterogeneous neoplastic disorders. They exhibit clonal proliferation of mature $\mathrm{B}, \mathrm{T}$, or natural killer (NK) lymphoid cells in the peripheral blood, bone marrow, lymph nodes, or other extranodal sites. They constitute $2.8 \%$ of all new cancer cases and $2.6 \%$ of mortality from malignancy worldwide [1]. The burden of NHL in India is estimated to be high with an incidence rate of 0.22 per million and a mortality rate of 0.15 per million population [2]. Diagnosis of CLPD has been a frequent challenge in 
hematology, and the flow cytometry (FCM) plays a very vital role for the screening as well as the specific identification and characterization of the expanded neoplastic lymphoid cells. The present World Health Organization (WHO) classification (2008 and the revised 2016 edition) has incorporated extensive description about the immunophenotype of neoplastic cells while describing the mature B, T, or NK lymphoid cell neoplasm or CLPDs [3].

The FCM is a powerful tool to analyze and characterize the heterogeneous mixed population of cells on a cell-by-cell basis. It provides a rapid, quantitative, and multi-parametric analysis. FCM is usually performed on cells in a liquid suspension being incubated with fluorescently labeled monoclonal antibodies (MoAb) directed against specific cellular proteins. The physical properties such as size and internal cellular complexity are being represented by forward angle light scatter (FSC) and rightangle scatter or side scatter (SSC), respectively. Combining FSC versus SSC can resolve certain cell population, e.g., mononuclear cells from polymorphonuclear cells. The use of multiple fluorochromes with overlapping excitation wavelengths but different emission wavelengths (or "colors") and different fluorescent (FL) detection channels can allow several cell properties to be measured simultaneously and thus achieves good discriminatory capacity. The relative fluorescence intensity of the positive cells provides a relative measure of antigen expression in the form of dim, moderate, or bright expression. Sometimes, the pattern of expression can be heterogeneous especially by the neoplastic cells [4].

Over the last five decades, FCM has been evolved from a research technique to a routinely used laboratory tool for the diagnosis, prognosis, and follow-up of neoplasm, immunodeficiency, or other varied disorders [5]. It has developed from a single parameter to multi-parameter assessment by use of multiple FL channels and fluorochromes in FCM. When a 4-color FCM is capable of measuring 6 parameters consisting of 2 scatters (forward scatter FSC and side scatter SSC) and 4 fluorescence parameters, the 8-color FCM can measure 10 parameters ( 2 scatter and 8 fluorescence). Similarly, the higher the number of colors, the higher is the number of parameter that can be analyzed simultaneously by the polychromatic flow cytometry [6].

Neoplasm of mature lymphoid cells lack antigenic features of immaturity, such as expression of terminal deoxynucleotidyl transferase (TdT), CD34, or weak CD45. They express lineageassociated markers with evidence of monoclonality. Depending upon the lineage-associated antigen expression, the neoplasm of mature lymphoid cells can be divided into those of B, T and NK cell lineages. Neoplasms of mature B lymphoid cells are usually distinguished from normal cells by the identification of the 2 main types of phenotypic abnormality: immunoglobulin light chain class restriction and aberrant antigen expression. Neoplasm of mature T and NK cells can often be identified by the flow cytometry immunophenotyping based on a combination of various findings. An abnormally increased number of T or NK lymphoid populations of cells form an indirect evidence of clonal proliferation which requires further detection of aberrant antigen expression or loss of expression of some pan markers. The deviation of the $\mathrm{CD} 4 / \mathrm{CD} 8$ ratio might be an indication of the presence of an abnormally restricted $\mathrm{T}$ population and warrants further evaluation to establish the neoplasm of T cells. It is important to recognize normal subsets of T cells, including T-cell receptor (TCR)- $\gamma / \delta^{+}$cells that may lack staining for CD5, CD4, and CD8. NK cells usually lack staining for $\mathrm{CD} 5$ and demonstrate variable expression for CD8. The deviation from the same can be the evidence of neoplasm [7, 8]. It is difficult to simultaneously analyze all the antibodies together to differentiate normal lymphocyte subset population, as well as identifying and differentiating the abnormal neoplastic population of cells. A multi-dimensional analysis by the highest color flow cytometry with the maximum possible antibody combination in a single panel may be an ideal option. But higher color comes with its own advantages and limitations.

A comparison between different flow cytometry models from 4- to 8-color combination of antibodies for screening suspected CLPD shows that panel designs having lower number of FL channels, and antibodies are limited by the number of parameters being analyzed simultaneously and hence the application too $[9,10]$. One tube antibody combination of all the informative primary markers by multiplexing more than one antibody in single channel is ideally the most valuable method for rapid evaluation (Table 1). The EuroFlow (EF) consortium had designed and evaluated different panels of clone of antibody and fluorophore combinations in different hematolymphoid neoplasm. They came up with a single tube immunophenotype (IPT) screening panel. The lymphoid screening tube (LST) consist of an 8-color, 12-marker combination of antibodies with an aim to detect the neoplasm of mature $\mathrm{B}, \mathrm{T}$, and $\mathrm{NK}$ cells in peripheral blood $(\mathrm{PB})$, bone marrow (BM), lymph nodes (LN), and different types of body fluids [11].

Hence, a study was undertaken with an objective to evaluate the diagnostic usefulness of a single LST FCM assay. It included 12 antibody cocktails in an 8-color labeling based on EF-proposed panel of antibodies with slight modifications of antibody and fluorophore combinations and a logical gating strategy to detect the clonal lymphoid cells for the diagnosis of CLPD. The definitive classification of mature lymphoid neoplasm was done by selection of further differentiating and definitive antibodies combinations.

\section{Material and methods}

This study was carried out in the Laboratory Hematology division from March 2017 to April 2019 to evaluate the diagnostic usefulness of a single LST FCM assay in all 


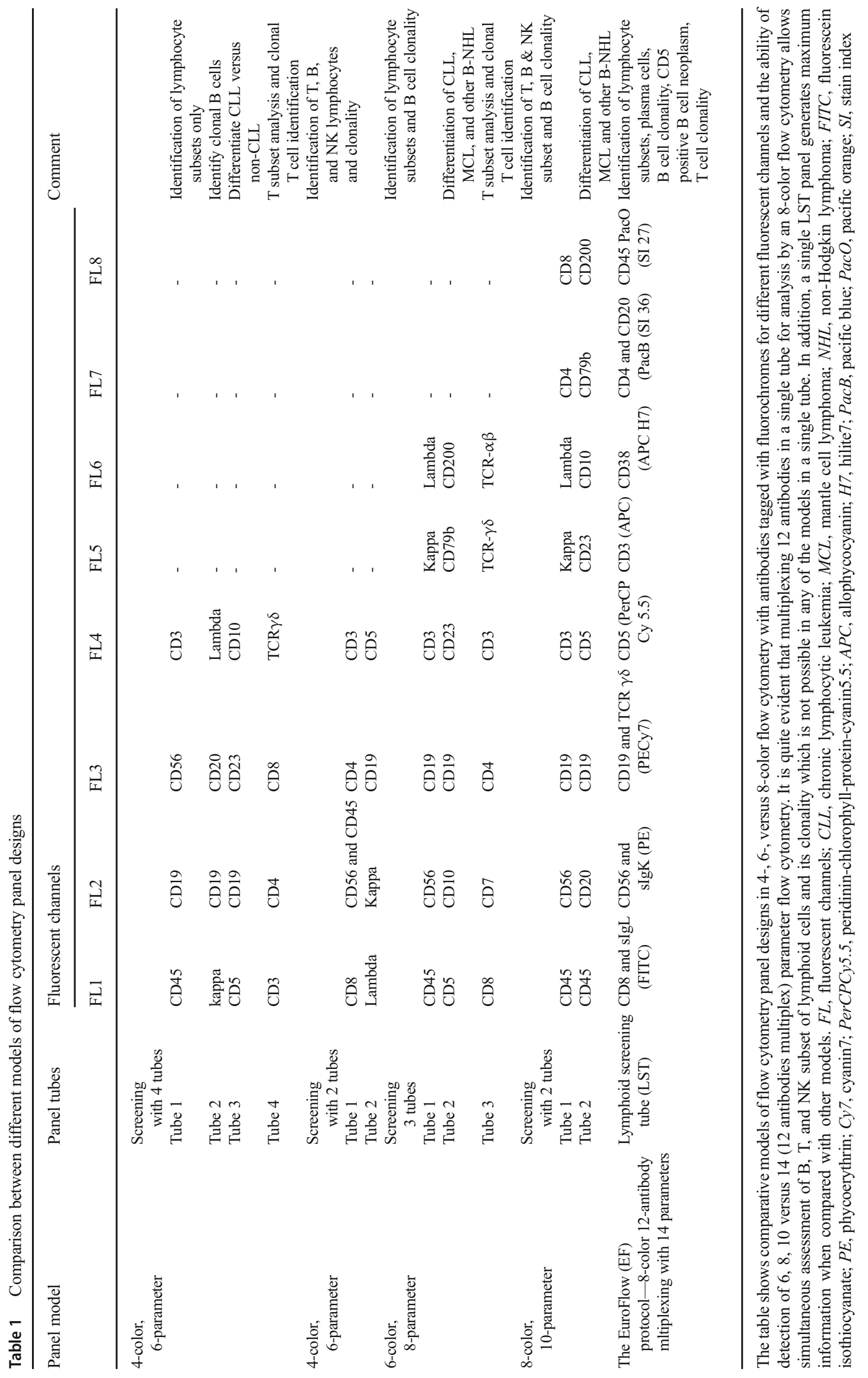


consecutive suspected CLPD cases. All the analysis were done using the BD FACSCanto ${ }^{\mathrm{TM}}$ II system with three lasers, a blue (488-nm, air-cooled, 20-mW solid state), a red (633$\mathrm{nm}, 17-\mathrm{mW} \mathrm{HeNe}$ ), and a violet (405-nm, 30-mW solid state), and 8 FL channels.

\section{Panel selection}

The panel for assessing CLPD was selected based upon the proposed EF panel of antibodies with some modifications and customization as per availability of antibodies [11, 12]. A primary LST was designed using 12 antibodies with an aim to identify the major subsets B, T, and NK lymphoid cells. CD45 was selected to differentiate mature versus immature lymphoid cells. The lymphoblasts express dim or negative CD45 whereas mature lymphocytes show bright CD45 expression with a low SSC. Further surface membrane, CD3 for the mature T cells, both CD19 and CD20 for the selection of B cells, CD56 for NK cells, and CD38 for plasma cells were included. The combination of CD19 and CD20 with CD45 would allow subsetting of $\mathrm{B}$ cells into mature $\mathrm{B}$ lymphocytes $\left(\mathrm{CD} 19^{+}, \mathrm{CD} 20^{\mathrm{hi}}\right.$, and $\left.\mathrm{CD} 45^{\mathrm{hi}}\right)$ and B cell precursors $\left(\mathrm{CD} 19^{+}\right.$, $\left.\mathrm{CD} 20^{-/ \mathrm{lo}}, \mathrm{CD} 45^{\mathrm{lo}}\right)$ too. NK cells should fulfill the criteria for mature lymphocytes $\left(\mathrm{CD} 45^{\text {hi }}, \mathrm{SSC}^{\mathrm{lo}}\right)$ in the absence of CD19 and surface membrane $\mathrm{CD} 3$ expression, and they would typically show reactivity for CD56. Additional markers selected for further subsetting of $\mathrm{B}, \mathrm{T}$, and NK cells and clonal proliferation included surface membrane immunoglobulin light chain kappa and lambda (sIgk and $\operatorname{sg} \lambda$ ) for B lymphocytes; $\mathrm{CD} 4, \mathrm{CD} 8$, and $\mathrm{T}$ cell receptor gamma-delta (TCR $\gamma \delta$ ) for T lymphocytes; and CD56 and CD8 for NK cells, respectively. The B lymphocytes are further looked for CD5 expression. It would have been valuable to include CD10 in the panel as the combined use of CD5 and CD10 is able to discriminate between different B-CLPD more efficiently. As our panel was a buildup of EF protocol justifying its use, hence, CD38 was preferred over CD10 in our panel too. CD38 is able to provide complimentary information about normal versus abnormal population of lymphocytes, precursor B cells, and plasma cells whereas CD10 has limitations in this regard. These antibody reagents were arranged in the LST by multiplexing the antibodies tagged with V450, V500, FITC, PE, PerCP$\mathrm{Cy}^{\mathrm{TM}}$ 5.5, $\mathrm{PE}-\mathrm{Cy}^{\mathrm{TM}} 7, \mathrm{APC}$, and APCH7 fluorochromes. We have included CD4 (clone SK3) and CD20 (clone L27) in V450 channel; CD8 (clone SK1) and surface membrane immunoglobulin kappa light chain (sIgK) (clone TB28-2) in FITC channel; CD56 (clone NCAM16) and surface membrane immunoglobulin lambda light chain $(\operatorname{sIg} \lambda)$ (clone 1155-2) in PE channel; and CD19 (clone SJ25-C1) and TCR- $\gamma \delta$ (clone 11F2) in PE-Cy ${ }^{\mathrm{TM}} 7$ channels. The two antibodies in the same channel were selected based upon the mutually exclusive expression of cells. Other antibodies used were CD45 V500-C (clone 2D1), surface membrane CD3
APC (clone SK7), CD38 APC-H7 (clone HB7), and CD5 PerCP-Cy ${ }^{\mathrm{TM}_{5}}$.5 (clone L17F12). All the MoAbs were from Becton Dickinson (BD) Biosciences (San Jose, CA, USA). Thus, an 8-color, 12-antibody, and 14-parameter single LST panel was designed compared with conventional 8-color and 10-parameter panels. The modifications in the proposed EF panel of antibodies applied in our panel were CD45 V500-C (stain index, SI 21), CD4 V450 (SI 42), and CD20 V450 instead of CD45 Pacific Orange (PacO) (SI 27), CD20 Pacific Blue (PacB) (SI 36), and CD4 PacB. All the mature $\mathrm{B}$ cell neoplasms with $\mathrm{CD} 5$ positivity or $\mathrm{CD} 5$ negativity were further analyzed to differentiate between chronic lymphocytic leukemia (CLL) versus non-CLL CLPD by using the combination of antibody of CD45, CD19, CD20, CD10, CD23, FMC-7, CD79b, CD43, and CD200. All B-CLPD cases found to be both $\mathrm{CD} 5$ and $\mathrm{CD} 10$ negative were further analyzed to differentiate hairy cell leukemia by combining CD45, CD19, CD103, CD11c, CD123, and CD25. All T cell CLPD cases were further analyzed by using CD45, CD3, CD7, CD10, CD25, CD2, CD30, HLA DR, and CD11c (Table 2).

\section{Sample collection}

After obtaining the informed consent from the patients or their relatives in all suspected cases of CLPD, a peripheral blood, bone marrow, or body fluid samples were collected in an EDTA or heparin anti-coagulated tube. The EDTA anticoagulated samples were analyzed within $24 \mathrm{~h}$ and the heparinized samples were analyzed within $48 \mathrm{~h}$ as per standard recommendations. A total leukocyte count of the sample was carried out on Sysmex® XN 1000 or 3000 (SYSMEX, Kobe, Japan) automated CBC and body fluid analyzer. A smear was stained by Leishman-Giemsa staining and microscopic evaluation was done in all cases to decide upon the selection of LST or acute leukemia panel based on morphological features. Further secondary panel of antibodies were selected based on evidence of clonality with combination of expression of various markers in the LST panel as well as microscopic morphology and other ancillary findings.

\section{Sample preparation and data acquisition}

All the samples were processed by the standard protocol of wash followed by stain, lyse, and wash for antibody staining. Then, 500,000 events were acquired in the flow cytometer BD FACSCanto $^{\text {TM }}$ II (BD Biosciences, San Jose, CA, USA) using the BD FACSDIVA ${ }^{\mathrm{TM}}$ software. The list mode data (LMD) file was stored for analysis and interpretation. Lesser events were acquired in some body fluid samples especially CSF. 
Table 2 The flow cytometry panel design of the lymphoid screening tube (LST) based on EuroFlow (EF) protocol with modifications and other secondary $\mathrm{B}$ and $\mathrm{T}$ tube panels for the evaluation of suspected CLPD

\begin{tabular}{|c|c|c|c|c|c|c|c|c|c|}
\hline \multirow{2}{*}{$\begin{array}{l}\text { Panel } \\
\text { design }\end{array}$} & \multicolumn{4}{|l|}{ Blue } & \multicolumn{2}{|l|}{ Red } & \multicolumn{2}{|l|}{ Violet } & \multirow[t]{2}{*}{ Comments } \\
\hline & FITC & $\mathrm{PE}$ & $\begin{array}{l}\text { PerCP } \\
\text { Cy } 5.5\end{array}$ & PECy7 & APC & APC H7 & $\begin{array}{l}\text { Horizon V450 } \\
\text { (SI 42) }\end{array}$ & $\begin{array}{l}\text { Horizon V500 } \\
\text { (SI 21) }\end{array}$ & \\
\hline Our LST & $\begin{array}{l}\text { CD8 and } \\
\text { sIgL }\end{array}$ & $\begin{array}{l}\text { CD56 and } \\
\text { sIgK }\end{array}$ & CD5 & $\begin{array}{r}\mathrm{CD} 19 \text { and } \\
\text { TCR } \gamma \delta\end{array}$ & smCD3 & CD38 & $\mathrm{CD} 4$ and $\mathrm{CD} 20$ & $\mathrm{CD} 45$ & $\begin{array}{l}\text { Identification of lymphocyte subsets } \\
\text { plasma cells, B cell clonality, } \\
\text { CD5 positive B cell neoplasm, } \\
\text { T cell clonality }\end{array}$ \\
\hline $\begin{array}{l}\text { Secondary B } \\
\text { tube } 1\end{array}$ & $\mathrm{CD} 23$ & CD200 & $\mathrm{CD} 79 \mathrm{~b}$ & CD19 & CD10 & CD43 & FMC-7 & $\mathrm{CD} 45$ & $\begin{array}{l}\text { To differentiate CLL versus } \\
\text { non-CLL CLPD }\end{array}$ \\
\hline $\begin{array}{l}\text { Secondary B } \\
\text { tube } 2\end{array}$ & $\mathrm{CD} 25$ & CD103 & $\mathrm{CD} 11 \mathrm{c}$ & CD19 & CD123 & - & - & CD45 & $\begin{array}{l}\text { To differentiate hairy cell leukemia } \\
\text { among CD5 and CD10 negative } \\
\text { CLPD }\end{array}$ \\
\hline $\begin{array}{l}\text { Secondary } \mathrm{T} \\
\text { tube } 1\end{array}$ & $\mathrm{CD} 25$ & $\mathrm{CD} 30$ & - & $\mathrm{CD} 2$ & $\mathrm{CD} 7$ & $\mathrm{CD} 3$ & HLA-DR & $\mathrm{CD} 45$ & $\begin{array}{l}\text { To differentiate different } \mathrm{T} \text { cells } \\
\quad \text { (limited markers were available }\end{array}$ \\
\hline $\begin{array}{l}\text { Secondary } \mathrm{T} \\
\text { tube } 2\end{array}$ & CD8 & CD16 & $\mathrm{CD} 11 \mathrm{c}$ & - & CD10 & $\mathrm{CD} 3$ & $\mathrm{CD} 4$ & CD45 & with us) \\
\hline
\end{tabular}

$F I T C$, fluorescein isothiocyanate; $P E$, phycoerythrin; $C y 7$, cyanin 7; $P e r C P C y 5.5$, peridinin-chlorophyll-protein-cyanin 5.5; $A P C$, allophycocyanin; $H 7$, hilite7; SI, stain index; $L S T$, lymphoid screening tube; $s$, surface; $I g$, immunoglobulin; $L$, lambda light chain; $K$, kappa light chain; TCR, T-cell rearrangement; $C L L$, chronic lymphocytic leukemia; $C L P D$, chronic lymphoproliferative disorder; $H L A-D R$, human leukocyte antigen-DR isotype

\section{Instrument set up}

BD FACSDiva ${ }^{\mathrm{TM}}$ CS\&T IVD beads were used on BD FACSCanto ${ }^{\mathrm{TM}}$ II flow cytometer running BD FACSDiva ${ }^{\mathrm{TM}}$ software to set up the cytometer, to perform daily performance quality control (QC), and to determine lyse/wash (LW) application settings. BD OneFlow Setup beads were used to set cytometer detector photomultiplier tube voltages (PMTVs) for the BD multicolor tube assay. Lyse washed blood (LWB) was used to set cytometer FSC and SSC voltages to a target value range. BD FC beads were used to calculate the fluorescence compensation matrix during setup of the BD FACSCanto ${ }^{\mathrm{TM}}$ II flow cytometer. Post-acquisition compensation setup was used wherever required during analysis. Reagent titration was done to get appropriate signal to noise ratio and the same dilution was used for sample processing [12].

\section{Analysis}

The LMD files were analyzed by the BD FACSDIVA ${ }^{\mathrm{TM}}$ software. The singlets were separated from doublets or triplets by using FSC height versus FSC area gating. The granulocytes, monocytes, lymphocytes, eosinophils, NRBC, and blast population were separately gated depending on the differential expression of CD45 versus SSC. A template was generated by analyzing 10 normal peripheral blood samples. The lymphocyte population showing bright CD45 with low SSC was analyzed by sequential gating. The $\mathrm{T}$ cells are gated using the positive expression of $\mathrm{CD} 3$. Then, a function of invert gating was used to separate non-T lymphocytes. The expression of CD19 is used to separate B cells followed by invert gating to separate non-B cells. The CD56 expression was used to separate NK cells. The bright CD38 expression was used to separate plasma cells among the non-B and non-T cells. The $\mathrm{T}$ cells were further analyzed to look for expression of TCR $\gamma \delta$, CD4, CD8, double positive for CD4 and CD8, and double negative for $\mathrm{CD} 4$ and $\mathrm{CD} 8$. The $\mathrm{B}$ cells were further analyzed to look for light chain expression and restriction, CD5 expression, CD20 expression, and CD38 expression. The NK cells are analyzed for CD8 expression. Figure 1 highlights the sequential gating and invert gating strategy used for analysis and interpretation. Further characterization and definitive classification of mature lymphoid neoplasm were done by using secondary panel of antibodies as discussed earlier.

The mature B cells were classified based on expression of CD5 and CD10 and size of lymphoid cells. The CD5+ve and CD10-ve cases with CD23 positivity were classified as CLL and CD23 negativity were classified as non-CLL. Mantle cell lymphoma (MCL) was diagnosed based on variable phenotype not typical for CLL with bright CD20, surface immunoglobulins (Ig), and CD79b positivity. A cyclin-D1 IHC and/or cytogenetic or molecular genetics for $t(11 ; 14) / C C N D$ rearrangement were studied to establish the definitive diagnosis. Whenever there was a variable immunophenotype with heterogeneous expression with overlapping features between CLL and MCL, a possibility of prolymphocytic leukemia (PLL) was considered after carefully evaluating entities with prolymphocytic transformation and microscopic differential of prolymphocytes [13]. A diagnosis of atypical CLL (aCLL) was favored for those cases with atypical morphology and atypical immunophenotype with negative or dim CD23, negative or dim CD5, strong expression of CD20, and strong sIg or CD79b positivity. The expression of CD200 was also considered during analysis which has emerged as a powerful 


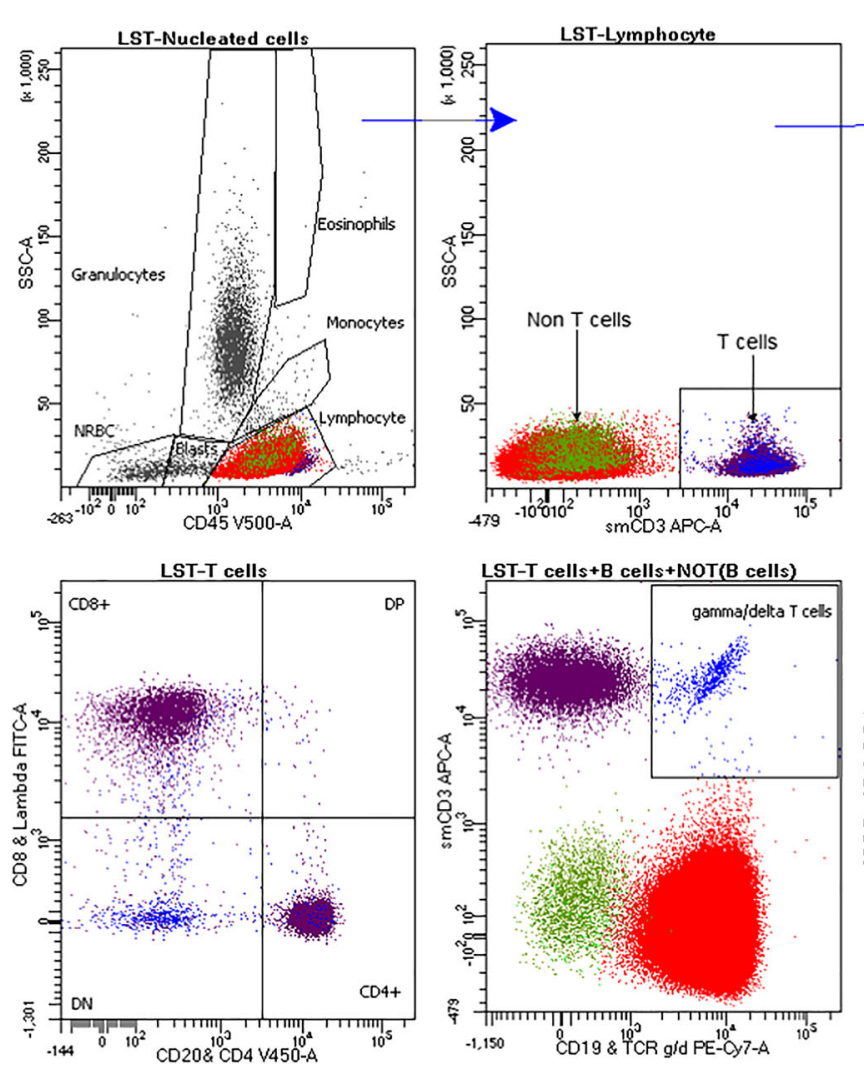

Fig. 1 Sequential and invert gating to separate different subset of leukocytes followed by lymphocytes which is further analyzed by subsetting it into T lymphocytes (CD $3+v e)$ and non T cells (CD $3-$ ve). The non T cells are further gated into B lymphocytes (CD $19+v e)$ and non-B lymphocytes. The non-B lymphocytes are further gated into NK lymphocytes (CD $56+v e$ ) and plasma cells (bright CD $38+v e$ )

marker to distinguish CLL from MCL. Of note, indolent leukemic MCL can be often positive for CD200 and some literatures claim that CD200 is often dimmer in atypical CLL [14]. Correlation with the microscopy morphology (large cells) or lymph node biopsy findings with IHC were considered for the diagnosis of diffuse large B cell lymphoma (DLBCL). All cases with CD5-ve and CD10+ve with small cell morphology and cleaving were further correlated with bone marrow biopsy, lymph node biopsy, and IHC (especially for bcl-2 and bcl-6 expression) before classifying as follicular lymphoma (FL). Cases with larger cells were further evaluated for Burkitt lymphoma (BL), DLBCL, etc. Lack of expression of CD5 and CD10 were further evaluated with hairy cell leukemia (HCL) markers CD103, CD123, CD25, and CD11c to classify hairy cell leukemia and its variants HCLv. Lack of hairy cell markers were diagnosed with marginal zone lymphoma (MZL) or lymphoplasmacytic lymphoma (LPL) depending on morphology, presence of clonal plasma cells and other laboratory investigations. Due consideration was given to evaluate the variable phenotypes of various CLPD for

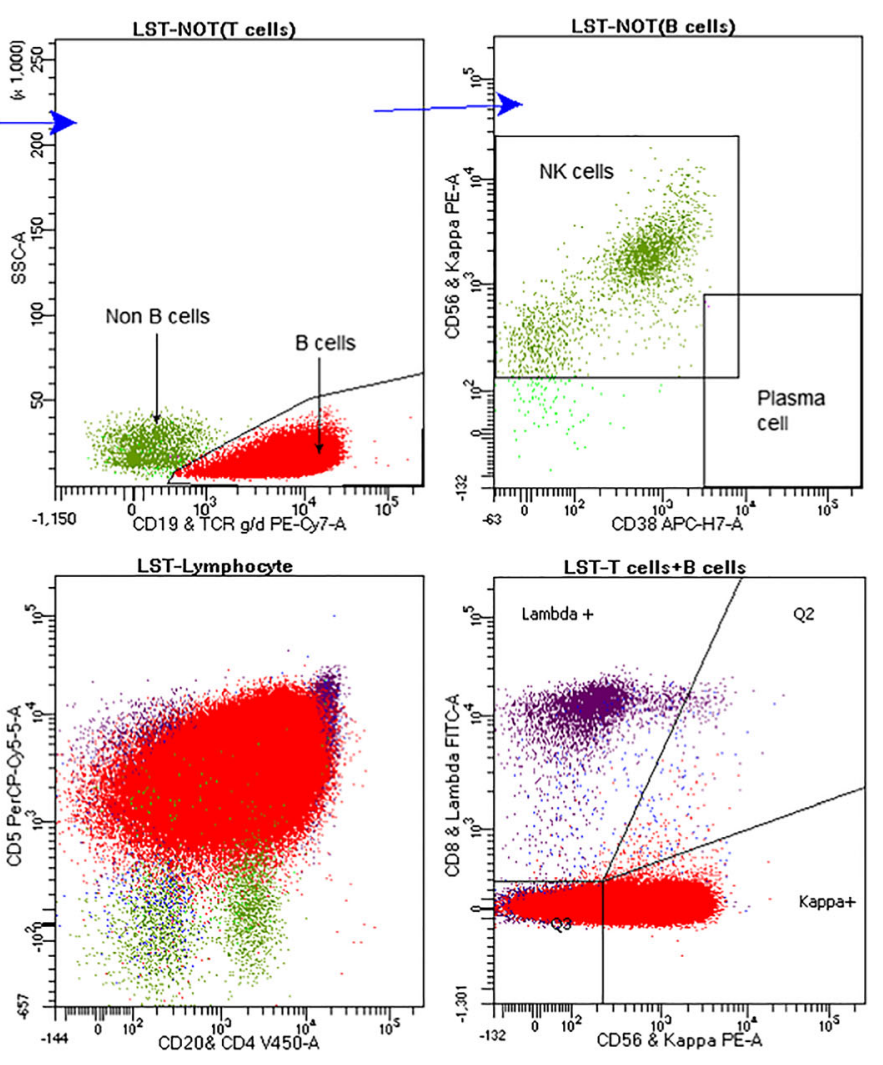

populations. T cell clonality was assessed by CD 4 and CD 8 and gamma-delta $\mathrm{T}$ cell expression pattern. The B cells are looked for monotypic light chain expression for clonality. The current case shows an example of B-CLPD with kappa restriction with CD5 moderate positivity and further evaluation established the diagnosis of chronic lymphocytic leukemia (CLL)

definitive diagnosis, e.g., CD5 and CD10 positive B cell lymphomas or $\mathrm{CD} 5 / \mathrm{CD} 10$ positive hairy cell leukemia.

Establishing clonality in suspected T-CLPD by flow cytometry is often difficult. The definitive demonstration of $\mathrm{T}$ cell clonality mostly rely upon the phenotypic analysis of the TCR repertoire, the identification of a clonal rearrangement of the T-cell receptor variable genes through molecular biology techniques (TCR gene rearrangement) or use of large number of antibodies to indicate aberrant gain or loss of antigenic expression. The use of TCR $\gamma \delta$, CD4, and CD8 in the LST can help in generating evidence in support of the presence of clonal $\mathrm{T}$ cells by disproportionate increase in a subset of $\mathrm{T}$ cells or abnormal CD4/CD8 ratio which further warrants evaluation to look for T cell clonality and hence T-CLPD. Due to lack of such sophisticated facility in our center, the feasibility of sending the sample to referral center for molecular analysis was also considered whenever required. Thus, $\mathrm{T}$ cells were evaluated for $\mathrm{TCR} \gamma \delta$ expression and expression of CD5, CD7, CD2, CD4, CD8, CD1a, CD10, CD25, CD30, and HLA DR to further classify peripheral $\mathrm{T}$ cell lymphomas $[3,7,10,11,15,16]$. 


\section{Statistical methods}

The data were collected and analyzed using the statistical software IBM SPSS 20 . The quantitative variables were summarized using mean $+/-$ SD for continuous variables with normal distribution and median with inter-quartile range (IQR) or 25th and 75th percentile in case of a skewed distribution. The non-parametric variables were analyzed by one-sample Kolmogorov-Smirnov test to look for normal distribution. The categorical variables were analyzed by frequency distribution. The diagnostic sensitivity and specificity of lymphoid screening tube in differentiating reactive versus neoplasm were also calculated and analyzed.

\section{Results}

We have analyzed 82 suspicious cases of lymphoma by a 3laser 8-color flow cytometry. The gender distribution was $76.8 \%(n=63)$ male and $23.2 \%(n=19)$ female with a male and female ratio 3.32:1. The age distribution ranged from minimum 15 years of age to maximum 81 years of age with mean age of 58.3 years (SD 12.7 years).

The type of specimen analyzed included peripheral blood $69.5 \%(n=57)$, bone marrow $23.2 \%(n=19)$, ascitic fluid $1.2 \%(n=1)$, pleural fluid $3.7 \%(n=3)$, and CSF $2.4 \%(n=2)$. The total leukocyte count of the samples varied from $0.92 \times$ $10^{3} / \mu \mathrm{L}$ to $635.62 \times 10^{3} / \mu \mathrm{L}$ with median count of $34.28 \times 10^{3} /$ $\mu \mathrm{L}$ (25th percentile, $17.51 \times 10^{3} / \mu \mathrm{L}$; and 75 th percentile, $\left.80.755 \times 10^{3} / \mu \mathrm{L}\right)$.

Out of 82 cases, 7 cases (8.5\%) had polyclonal population of $\mathrm{B}$ and T cells. They did not elicit any specific clonality of T, B, or NK lymphoid cells. Two cases $(2.4 \%)$ were showing dim expression of CD45 with markers of immaturity on secondary tubes, suggestive of acute lymphoblastic leukemia (ALL). A total of 73 cases (89\%) showed evidence of monoclonality of B or T cells suggestive of non-Hodgkin lymphoma (NHL). Out of which $97.3 \%(n=71)$ were classified as mature B cell neoplasm (B-NHL) and $2.7 \%(n=2)$ were mature $\mathrm{T}$ cell neoplasm (T-NHL). The use of further secondary IPT lymphoid markers, IHC, and/or molecular or cytogenetics, we could establish the diagnosis as CLL 59.2\% $(n=42)$, MCL $14.1 \%(n=10)$, follicular lymphoma (FL) $4.2 \%(n=3)$, LPL $4.2 \%(n=3)$, HCL $4.2 \%(n=3)$, and DLBCL $2.8 \%(n=2)$. The chronic lymphocytic leukemia/ prolymphocytic leukemia (CLL/PLL), MZL, monoclonal B cell lymphocytosis (MBL), and Burkitt's lymphoma (BL) constituted $1.4 \%(n=1)$ each. But a definitive diagnosis could not be established in $5.6 \%(n=4)$ of cases in view of lack of further work up due to refusal or referral. The frequency distribution is depicted in Fig. 2.

The monoclonality could be established on the gated B cells whose count varied from $99.0 \%$ to as low as $14.70 \%$ of all lymphoid cells with mean levels of $79.5 \%( \pm 18.75)$. When we compared the surface membrane immunoglobulin (Ig) kappa or lambda light chain expression in B-NHL, 56.3\% (n $=40$ ) showed expression of monotypic kappa light chain whereas $36.6 \%(n=26)$ showed monotypic lambda light chain. There were $7.1 \%(n=5)$ B-NHL which showed lack of both kappa and lambda light chain expression. The pattern of light chain expression of CLL $(n=42)$ showed $42.9 \%(n=$ 18) monotypic surface kappa expression and $45.2 \%(n=19)$ monotypic surface lambda expression whereas $11.9 \%(n=5)$ cases showed absence of light chain expression. The MCL ( $n$ $=10)$ showed majorly kappa positivity $90 \%(n=9)$ compared with lambda positivity $10 \%(n=1)$. Surface kappa was positive in $33.3 \%(n=1)$ of HCL $(n=3)$ and surface lambda was positive in $66.7 \%(n=2)$ cases. The FL showed $33.3 \%(n=1)$ cases to be kappa restricted and $66.7 \%(n=2)$ to be lambda light chain restricted. All the LPL cases $(n=3)$ were showing monotypic kappa expression. The DLBCL $(n=2)$ cases were showing kappa and lambda light chain restriction in one case each. The one MBL case was showing monotypic kappa expression. The one CLL/PLL case was showing monotypic kappa expression. The lone MZL was showing kappa light chain restriction. The BL was showing kappa restriction too.

The use of CD5 surface expression helped too in indicating clonality as a very small proportion of normal B cells is CD5 positive. The B-NHL showed $74.6 \%(n=53) \mathrm{CD} 5$ positive BCLPD and $25.4 \%(n=18)$ CD5 negative B-CLPD. The CD5 positive cases were further classified depending upon additional findings to be CLL 79.2\% ( $n=42)$, MCL $17.0 \%$ ( $n=$ 9), MBL $1.9 \%(n=1)$, and CLL/PLL $1.9 \%(n=1)$. In the CD5 negative cases, the clonality was established by combining the monotypic light chain expression with it. They were further diagnosed to be HCL $16.7 \%(n=3)$, FL $16.7 \%(n=3)$, LPL $16.7 \%(n=3)$, DLBCL $11.1 \%(n=2)$, BL $5.6 \%(n=1)$, and MZL $5.6 \%(n=1)$. Only one case of mantle cell lymphoma was found to be $\mathrm{CD} 5$ negative.

When we compared the expression pattern of CD20 positivity among the B-NHL, we found that most $69 \%(n=29)$ of CLL cases showed dim expression of CD20 with some showing heterogeneous expression, i.e., negative to dim or moderate positive. All other B-CLPD, i.e., non-CLL CLPD, showed moderate or bright expression of CD20. Similarly, CLL showed dim expression of light chain in comparison to nonCLL CLPD. (Fig. 3)

The 2 cases of T-NHL were showing leukocytosis with atypical lymphoid cells with $\mathrm{T}$ cell predominance on flow cytometry. T lymphocyte subsets showed predominantly CD4 and CD8 negative (double negative) cells (> 90\%) with $78.40 \%$ and $87.00 \%$ of TCR- $\gamma \delta$ expression, respectively. Hence, a diagnosis of $\gamma \delta \mathrm{T}$ cell lymphoma was favored in them.

Rest of the cases showed normal proportions of T subset of cells. The mature B-NHL showed proportion of $9.3 \%$ (25th percentile, 5.0\%; and 75th percentile, 19.7\%) $\mathrm{T}$ cells of all 


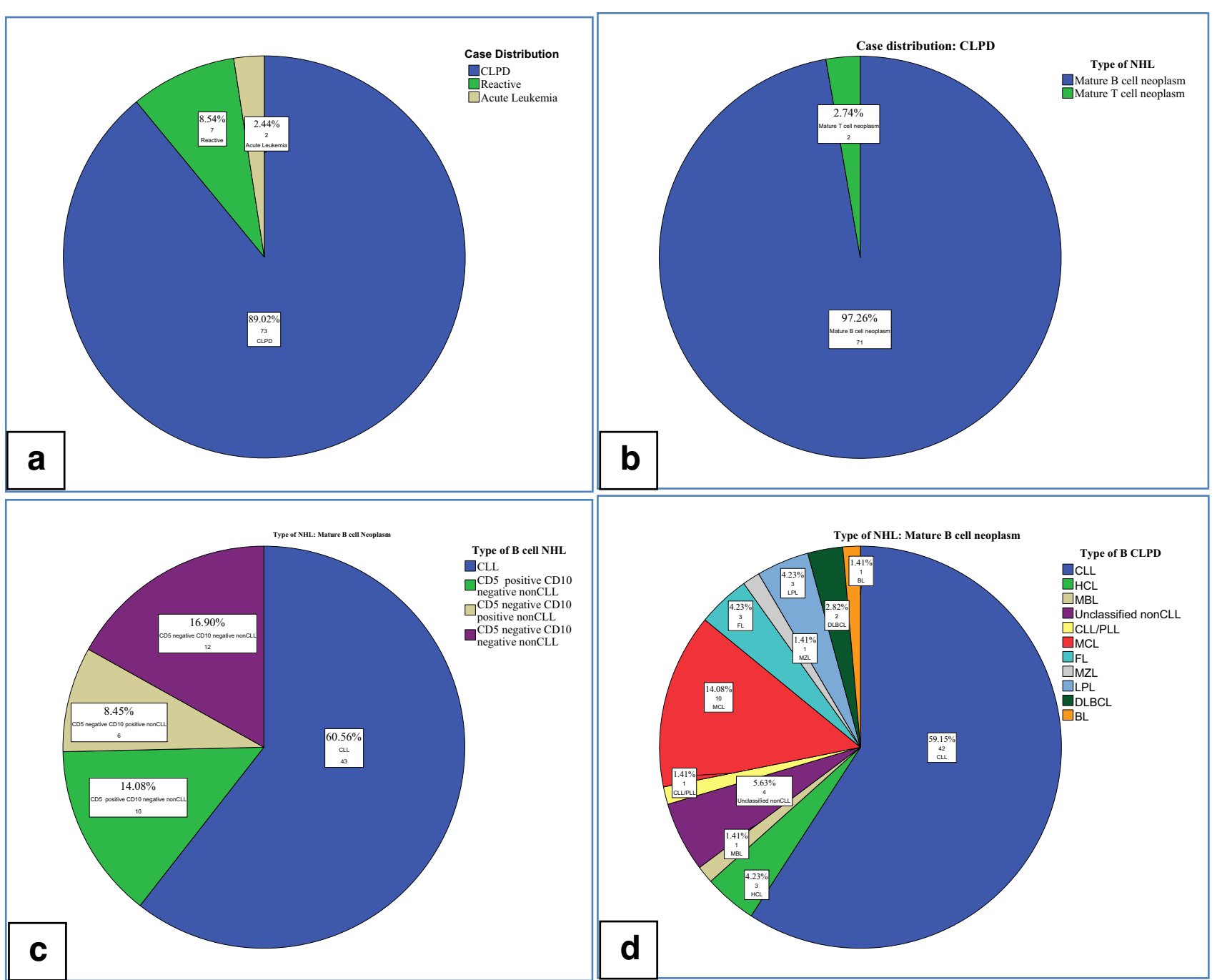

Fig. 2 Frequency distribution of various suspected CLPD cases analyzed by flow cytometry. a Broad classification into reactive, acute leukemia, or CLPD based on immunophenotypic findings. b Distribution into B, T, or NK CLPD among all CLPD cases. c Distribution of various broad categories of mature B cell neoplasm CLL and non-CLL CLPD. NonCLL CLPD is further classified based on CD5 and CD10 expression. $\mathbf{d}$ Distribution of various types of mature B cell neoplasms. Abbreviation:
CLPD, chronic lymphoproliferative disorder; Unclassi, Unclassifiable; CLL, chronic lymphocytic leukemia; HCL, hairy cell leukemia; MBL, monoclonal B lymphocytosis; PLL, prolymphocytic leukemia; MZL, marginal zone lymphoma; MCL, mantle cell lymphoma; FL, follicular lymphoma; LPL, lymphoplasmacytic lymphoma; DLBCL, diffuse large B cell lymphoma; BL, Burkitt lymphoma. lymphoid cells. The T cells showed $53.2 \%( \pm 12.4)$ of CD4+ T cells and $38.2 \%( \pm 11.1)$ of CD8+ T cells with a CD4/CD8 ratio $1.6( \pm 0.8)$.

The proportions of large cells were calculated by flow cytometry based on the comparison with the FSC of granulocytes which was taken as a positive reference population. The granulocytes were gated by SSC versus CD45. The CLL, MBL, FL, LPL, and CLL/PLL showed small proportion of large cells in comparison to other lymphomas. Mantle cell lymphoma showed mixed population of cells whereas diffuse large B cell lymphoma, Burkitt's lymphoma, and hairy cell leukemia showed proportionately higher number of larger cells as evident from the box plot (Fig. 4).
We analyzed the total number of tubes used for the diagnosis of different cases. The 7 cases $(8.5 \%)$ having polyclonal population of $\mathrm{B}$ cells and $\mathrm{T}$ cells did not require further analysis, even there was a good correlation with the clinical findings as well as other ancillary laboratory investigations. Besides, they did not elicit any evidence of mature lymphoid neoplasm. The 2 cases (2.4\%) showing dim expression of CD45 on LST were further analyzed as per our laboratory protocol for acute lymphoblastic leukemia (ALL) with additional one tube and 8 antibodies. A total of 73 cases (89\%) showed evidence of monoclonality of B or T cells suggestive of NHL requiring further analysis by flow cytometry. Out of the 71 cases $(97.3 \%)$ of mature B cell neoplasm (B-NHL), 

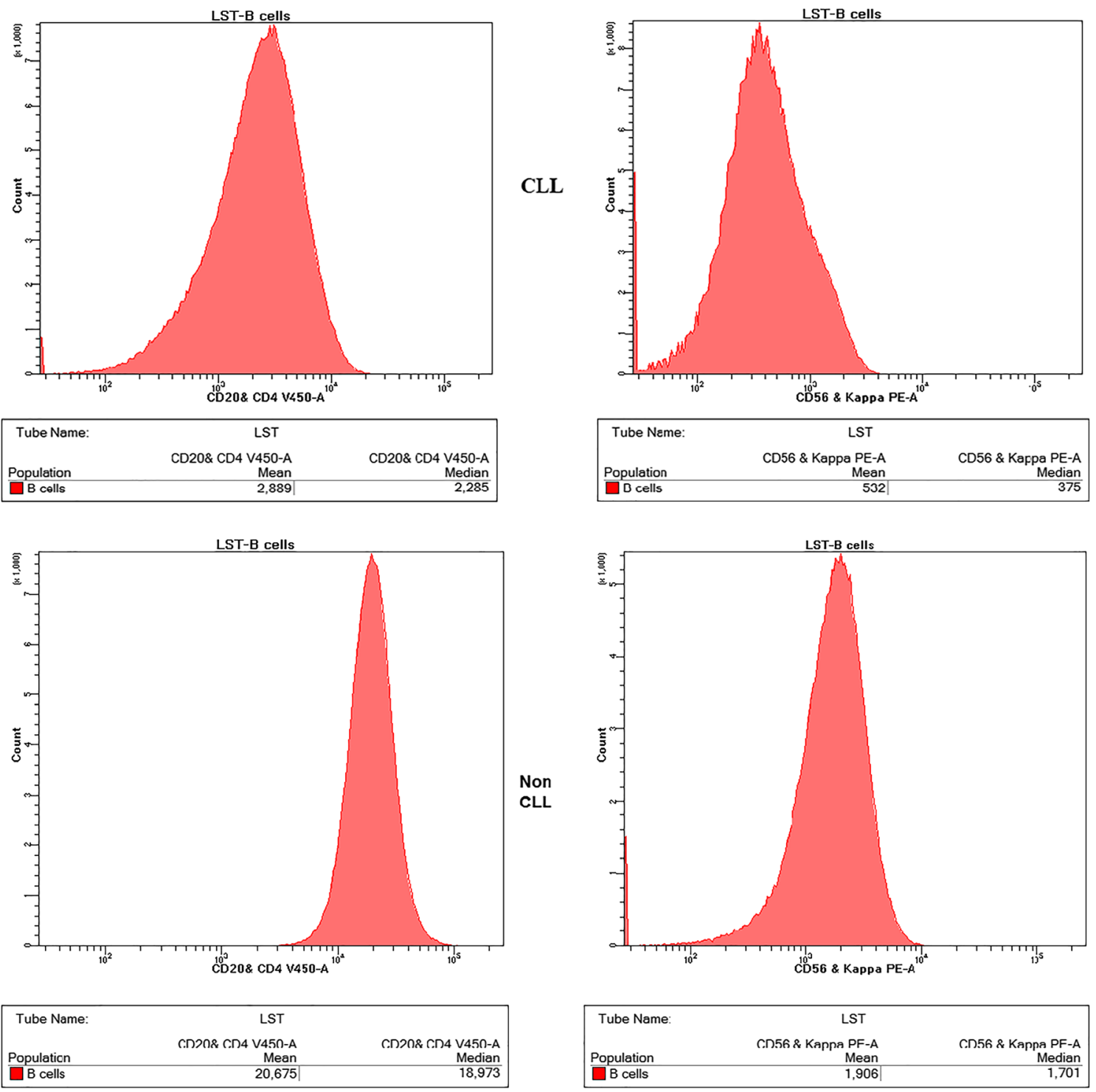

Fig. 3 Histogram shows common pattern of expression of CD20 and surface immunoglobulin light chain of CLL versus non-CLL CLPD. CLL shows weak or dim CD20 with weak and monotypic dim surface light chain (kappa expression in the present figure) in comparison to non-CLL CLPD

only 12 cases $(16.9 \%)$ required 2 secondary tubes (total 3 tubes and 27 antibodies) whereas a diagnosis could be inferred from the use of only one additional secondary tube for all other cases (total 2 tubes and 20 antibodies). All those 12 cases required 3 tubes because they were both $\mathrm{CD} 5$ and $\mathrm{CD} 10$ negative B-NHL and it was necessary to rule out hairy cell leukemia or its variants specifically. We did not require any further antibodies in those 2 cases of mature T cell neoplasm ( $\mathrm{T}$ NHL) as they were established to be $\gamma \delta$-T cell NHL. Thus, the total number of tubes used for 82 cases were 167 instead of 246 tubes which might have been required when a standard protocol of 3 screening tubes was followed in all suspected cases of CLPD. Similarly, total number of antibodies used was 1652 instead of 2214.
The diagnostic utility of lymphoid screening tube (LST) in differentiating reactive versus lymphoid neoplasm was found to have $100 \%$ sensitivity in our study.

\section{Discussion}

Flow cytometry evaluates individual cells in fluid suspension for the presence or absence of specific cellular antigens (phenotype). It has become an essential tool in the assessment for hematological malignancies. It helps in identifying and delineating cells from different lineages, stage of maturity, clonality, and synchronous or asynchronous expression in 


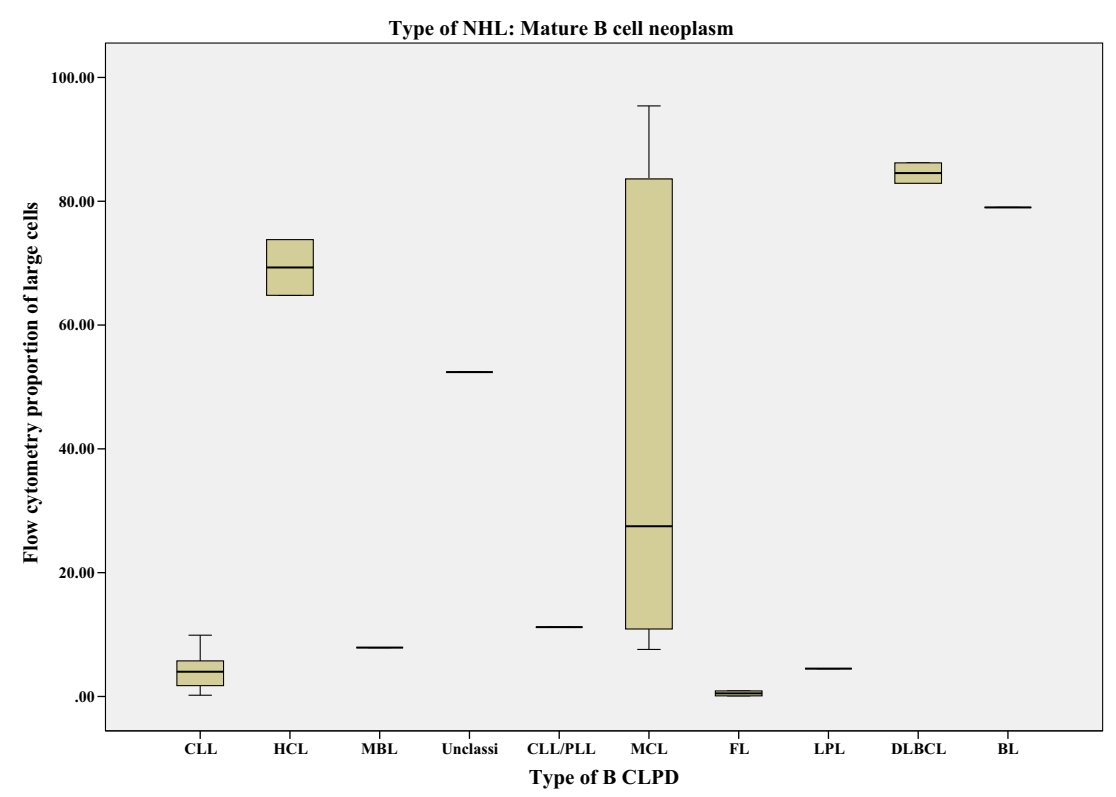

Fig. 4 Shows the box plot showing the distribution of different types of non-Hodgkin lymphoma (NHL) with flow cytometric proportion of large cells. CLL, MBL, FL, LPL, and CLL/PLL showed small proportion of large cells in comparison to other lymphoma. HCL, BL, and DLBCL show large proportion of large cells. Abbreviation: CLPD, chronic lymphoproliferative disorder; Unclassi, unclassifiable; CLL, chronic lymphocytic leukemia; HCL, hairy cell leukemia; MBL, monoclonal B lymphocytosis; PLL, prolymphocytic leukemia; MCL, mantle cell lymphoma; FL, follicular lymphoma; LPL, lymphoplasmacytic lymphoma; DLBCL, diffuse large B cell lymphoma; BL, Burkitt lymphoma comparison to normal population of cells. It also helps in deciding on further additional studies that might be of diagnostic value such as immunohistochemistry (IHC), conventional cytogenetic, fluorescence in situ hybridization (FISH), and molecular assays [7, 17].

One needs to combine immunophenotype markers to design the FCM panel in order to answer one or multiple relevant clinical questions through the identification, enumeration, and characterization of the relevant cell populations in the sample. Each case may require a different strategy to identify the target cell population. A rapid screening step based on a limited number of antibodies (preferably in a single tube) directed at differential identification of all relevant lymphoid cell subsets in the sample is generally most efficient. The British Committee for Standards in Haematology (BCSH) recommended the first line monoclonal antibodies consisting of CD19, CD23, FMC7, sIg (kappa and lambda), CD22, CD79b, CD2, and CD5 followed by second line markers for CLPD in 2002. In view of some proportion of T-CLPD being CD3 negative, CD2 was preferred instead of CD3 [18]. In 2006, the Latin American consensus panel recommended minimum 7 antibodies against CD3, CD19, CD56, CD4, CD8, kappa, and lambda light chains followed by other markers to classify or confirm CLPD [10]. An Indian consensus guidelines recommended a primary flow cytometry panel with minimum 9 antibodies including CD3, CD19, CD20, CD5, CD10, CD23, FMC7, kappa, and lambda light chains [19]. Similarly, the 2006 Bethesda international consensus included CD45, CD19, CD20, CD5, CD10, kappa, lambda,
$\mathrm{CD} 2, \mathrm{CD} 3, \mathrm{CD} 4, \mathrm{CD} 8, \mathrm{CD} 7$, and $\mathrm{CD} 56$ as primary reagents in the evaluation of suspected B, T, or NK neoplasm [20]. The EF consortium evaluated sequentially different 8-color antibody panels and fluorochrome combinations in the diagnosis of hematolymphoid neoplasm and came up with the guidelines for instrument settings, sample processing, and flow cytometry protocols. They proposed a lymphoid screening tube consisting of 12 MoAbs combination in a 8-color flow cytometry setup [11, 12]. Similarly, the GBCFLUX CLPD Committee had proposed a single screening panel consisting of 4-color 7-antibody multiplex to identify B, T, and NK cells [9]. But it lacks CD45 (leukocyte common antigen, LCA) which has the disadvantage of inability to identify immature cells as well as differentiating CD5 positive or negative CLPD.

We have selected a modified EF as a single LST and found a sensitivity of $100 \%$ which is comparable to similar sensitivity by the EuroFlow participating laboratories. They have found that the LST detected aberrant $\mathrm{B}$, T, or NK cell immunophenotypes in 149/150 (99.4\%) of B-CLPD and in $78 / 83(94 \%)$ of T/NK-CLPD, with an overall frequency of $97.4 \%[11]$.

In our study, we have analyzed 82 cases of suspected CLPD with a male: female ratio of 3.3:1 and mean age of 58 years with age range of 15 to 81 years. Out of which 73 cases were established to be CLPD. The mean age of presentation for CLPD was 59.2 years (SD 11.8) with age range 15 to 81 years and the male to female ratio observed was 3.3:1. Similar demographics have been reported by Geeta V Patil et.al., 
where they have analyzed a total of 66 cases of CLPD, out of which six (9\%) were $\geq 50$ years, $40(60.6 \%)$ were between 51 and 70 years and $20(30.3 \%)$ were $>70$ years. The overall median age reported was 64.5 years and the age range was from 43 to 84 years with a male to female ratio of 2.3:1 [21].

Among all 82 cases of suspected CLPD analyzed, 7 cases $(8.5 \%)$ were found to have polyclonal population of lymphoid cells or no abnormal population, $2(2.4 \%)$ cases as acute lymphoblastic leukemia and 73 (89\%) as CLPD. Out of which majority of CLPD was constituted by B-CLPD $97.3 \%$ with only 2 cases $(2.7 \%)$ as T-CLPD. Unfortunately, we did not encounter any NK CLPD cases during our study. Out of BCLPD cases, CLL was the most commonly encountered CLPD with a frequency of $59.2 \%$ followed by MCL $14.1 \%$. Other variants were less common as far as peripheral blood or bone marrow flow cytometry was concerned. Similar findings have been reported by Geeta V Patil et al. [21].

The current panel could able to simultaneously analyze 14 parameters of a cell by multiplexing 12 antibodies in an 8color flow cytometry setup. It had the advantage of delineating the B, T, and NK cell subsets of lymphocytes, its pattern of maturity, and identification of clonality of cells in all suspicious CLPD cases. In comparison, a panel being used in 4color, 6-color, or 8-color flow cytometry will require higher number of tubes as well as higher number of antibodies considering the fact that the backbone markers like CD45, CD19, or $\mathrm{CD} 3$ need to be maintained in all tubes, and hence, it adds to the cost, man power, and time consumption. Though 8 colors with 12 antibodies have other limitations as far as compensation and standardization are concerned, in a wellstandardized laboratory, it is more cost-effective method for investigating suspicious CLPD cases with higher diagnostic utility. The current LST panel is advantageous in many ways. It can differentiate $\mathrm{CD} 5$ positive versus negative CLPD. In addition, one can differentiate CLL by the combination of $\operatorname{dim}$ CD20, dim or negative immunoglobulin light chain with CD5 expression in many cases, though the importance of further markers like CD10, CD23, CD79b, FMC7, CD200, CD43, CD81, and ROR1 cannot be neglected [22, 23]. It has also the advantage of establishing clonality even though the proportion of neoplastic cells is low. It is of interest to note that the majority of MCL cases in our study showed kappa light chain restriction. Other studies have shown that MCL more often expresses lambda light chain when compared with other B cell lymphomas [24].

We also wish to emphasize that the use of LST can reduce the total antibody use, total tubes run, time on the analysis, cost to patients, and the healthcare system. An algorithmic approach should be followed for the selection of the secondary tubes or panel of antibodies which is dependent on the pattern of expression of lymphoid cells in LST. When both the primary and secondary panels of antibodies are processed simultaneously, it adds to the total expenditure. We could actually save nearly one third of the total cost due to reduced number of tubes as well as antibodies. It is evident that the total number of tubes reduced from 3 to 2.03 . The average number of antibodies used also reduces to 20.14 in comparison to 28 antibodies required as per standard protocol. Though the total time spent on processing is felt to be marginally high, we can bring down the total cost per reportable test of flow cytometry by 20 percentages considering $32.2 \%$ savings on antibody and adding $10 \%$ for manpower, consumables, and other ancillary expenditures. This helped us to keep our tariff also low where we receive many patients from lower and middle socioeconomic status.

In developing countries, affordability of patients has been always a challenge. A single screening panel with microscopic assessment can be less expensive solution for differentiating neoplasm versus reactive condition as well as guiding the therapy. It also reduces the total number of MoAbs used and thus reduces cost per test too. We have also found that flow cytometric proportion of large cells with granulocyte FSC as reference has additional diagnostic value in differentiating different NHL.

The present study is limited by its smaller sample size and very small number of T-CLPD (just 2 cases) and absence of any NK CLPD cases to analyze and get more valid evidence. The ability to detect more unusual cases needs to be further evaluated through a larger multi-centric study. However, multiplexing 12 antibodies in a LST for analysis on an 8color flow cytometry is diagnostically more useful and costeffective method.

\section{Compliance with ethical standards}

Conflict of interest The authors declare that they have no conflict of interest.

\section{References}

1. Bray F, Ferlay J, Soerjomataram I, Siegel RL, Torre LA, Jemal A (2018) Global cancer statistics 2018: GLOBOCAN estimates of incidence and mortality worldwide for 36 cancers in 185 countries, CA. Cancer J Clin 68(6):394-424

2. Nair R, Arora N, Mallath MK (2016) Epidemiology of nonHodgkin's lymphoma in India. Oncology 91(1):18-25

3. Swerdlow SH et al (2017) WHO classification of tumors of haematopoietic and lymphoid tissues, revised 4th edition. International agency for research on cancer (IARC), Lyon

4. Brown M, Wittwer C (2000) Flow cytometry: principles and clinical applications in hematology. Clin Chem 46(8):1221-1229

5. Jaye DL, Bray RA, Gebel HM, Harris WAC, Waller EK (2012) Translational applications of flow cytometry in clinical practice. J Immunol 188(10):4715-4719

6. Rosa SCD, Brenchley JM, Roederer M (2003) Beyond six colors: a new era in flow cytometry. Nat Med 9(1):112-117

7. Craig FE, Foon KA (2008) Flow cytometric immunophenotyping for hematologic neoplasms. Blood 111(8):3941-3967 
8. Stetler-Stevenson M, Davis B, Wood B, Braylan R (2007) 2006 Bethesda International Consensus Conference on flow cytometric immunophenotyping of hematolymphoid neoplasia. Cytometry B Clin Cytom 72B(S1):S3-S3

9. Sales MM, Ferreira SIACP, Ikoma MRV, Sandes AF, Beltrame MP, Bacal NS et al (2017) Diagnosis of chronic lymphoproliferative disorders by flow cytometry using four-color combinations for immunophenotyping: a proposal of the Brazilian group of flow cytometry (GBCFLUX). Cytometry B Clin Cytom 92(5):398-410

10. Ruiz-Argüelles A, Rivadeneyra-Espinoza L, Duque RE, Orfao A (2006) Report on the second Latin American consensus conference for flow cytometric immunophenotyping of hematological malignancies. Cytometry B Clin Cytom 70B(1):39-44

11. van Dongen JJM, Lhermitte L, Böttcher S, Almeida J, van der Velden VHJ, Flores-Montero J, Rawstron A, Asnafi V, Lécrevisse Q, Lucio P, Mejstrikova E, Szczepański T, Kalina T, de Tute R, Brüggemann M, Sedek L, Cullen M, Langerak AW, Mendonça A, Macintyre E, Martin-Ayuso M, Hrusak O, Vidriales MB, Orfao A, EuroFlow Consortium (EU-FP6, LSHB-CT-2006018708) (2012) EuroFlow antibody panels for standardized $n$-dimensional flow cytometric immunophenotyping of normal, reactive and malignant leukocytes. Leukemia 26(9):1908-1975

12. Kalina T, Flores-Montero J, van der Velden VHJ, Martin-Ayuso M, Böttcher S, Ritgen M, Almeida J, Lhermitte L, Asnafi V, Mendonça A, de Tute R, Cullen M, Sedek L, Vidriales MB, Pérez JJ, te Marvelde JG, Mejstrikova E, Hrusak O, Szczepański T, van Dongen J, Orfao A, EuroFlow Consortium (EU-FP6, LSHB-CT2006-018708) (2012) EuroFlow standardization of flow cytometer instrument settings and immunophenotyping protocols. Leukemia 26(9): 1986-2010

13. van der Velden VHJ, Hoogeveen PG, de Ridder D, der Struijk MS, van Zelm MC, Sanders M et al (2014) B-cell prolymphocytic leukemia: a specific subgroup of mantle cell lymphoma. Blood 124(3): 412-419

14. Sandes AF, Chauffaille M d L, Oliveira CMC, Maekawa Y, Tamashiro N, Takao TT et al (2014) CD200 has an important role in the differential diagnosis of mature B-cell neoplasms by multiparameter flow cytometry. Cytometry B Clin Cytom 86(2):98-105

15. Davis BH, Foucar K, Szczarkowski W, Ball E, Witzig T, Foon KA, Wells D, Kotylo P, Johnson R, Hanson C, Bessman D (1997) U.S.Canadian consensus recommendations on the immunophenotypic analysis of hematologic neoplasia by flow cytometry: Medical indications. Cytometry 30(5):249-263

16. Mahe E, Pugh T, Kamel-Reid S (2018) T cell clonality assessment: past, present and future. J Clin Pathol 71(3):195-200

17. Philippé J, Nollet F, Bakkus M, Meeus P, Demanet C, SchaafLafontaine N, Franke S, Chatelain B, Vermeulen K, Boone E, El
Housni H, Heimann P, Husson B, Lambert F, Vannuffel P, Saussoy P, Maes B, Deschouwer P (2009) Guidelines for an integrated diagnostic approach of chronic lymphoproliferative disorders in the routine laboratory of haematology in belgium. Acta Clin Belg 64(6):494-504. https://doi.org/10.1179/acb.2009.085

18. Bain BJ, Barnett D, Linch D, Matutes E, Reilly JT, General Haematology Task Force of the British Committee for Standards in Haematology (BCSH), British Society of Haematology (2002) Revised guideline on immunophenotyping in acute leukaemias and chronic lymphoproliferative disorders. Clin Lab Haematol 24(1):113

19. Gujral S, Subramanian PG, Patkar N, Badrinath Y, Kumar A, Tembhare P, Vazifdar A, Khodaiji S, Madkaikar M, Ghosh K, Yargop M, Dasgupta A (2008) Report of proceedings of the national meeting on "Guidelines for Immunophenotyping of Hematolymphoid Neoplasms by Flow Cytometry". Indian J Pathol Microbiol 51(2):161

20. Wood BL, Arroz M, Barnett D, DiGiuseppe J, Greig B, Kussick SJ et al (2007) 2006 Bethesda International Consensus recommendations on the immunophenotypic analysis of hematolymphoid neoplasia by flow cytometry: optimal reagents and reporting for the flow cytometric diagnosis of hematopoietic neoplasia. Cytometry B Clin Cytom 72B(S1):S14-S22

21. Okaly GVP, Nargund AR, Venkataswamy E, Jayanna PK, Juvva CR, Prabhudesai S (2013) Chronic lymphoproliferative disorders at an Indian Tertiary Cancer Centre - the panel sufficiency in the diagnosis of chronic lymphocytic leukaemia. J Clin Diagn Res JCDR 7(7):1366-1371

22. Rawstron AC, Kreuzer K, Soosapilla A, Spacek M, Stehlikova O, Gambell P et al (2018) Reproducible diagnosis of chronic lymphocytic leukemia by flow cytometry: an European Research Initiative on CLL (ERIC) \& European Society for Clinical Cell Analysis (ESCCA) Harmonisation project. Cytometry B Clin Cytom 94(1): 121-128

23. Seegmiller AC, Hsi ED, Craig FE (2019) The current role of clinical flow cytometry in the evaluation of mature B-cell neoplasms. Cytometry B Clin Cytom. 96(1):20-29

24. Bertoni F, Zucca E, Genini D, Cazzaniga G, Roggero E, Ghielmini M, Cavalli F, Biondi A (1999) Immunoglobulin light chain kappa deletion rearrangement as a marker of clonality in mantle cell lymphoma. Leuk Lymphoma 36(1-2):147-150

Publisher's note Springer Nature remains neutral with regard to jurisdictional claims in published maps and institutional affiliations. 